\title{
Updates on HIV treatment and prevention from Asia's HIV symposium: the 18th Bangkok International Symposium on HIV Medicine
}

Pirapon J Ohata ${ }^{*}$, , Anchalee Avihingsanon ${ }^{1,2}$, Sasiwimol Ubolyam', Opass Putcharoen ${ }^{3}$, Stephen J Kerr', Alain Volnysanne', Kesdao Nanthapisal', Chavalun Ruengpayyathip', Torsak Bunupuradah', Wasana Prasitsuebsai', Sivaporn Kukanok', Tanya Do', Nadia K Landolt', Kiat Ruxrungtham ${ }^{1,2}$, Praphan Phanuphak ${ }^{1,5} \&$ on behalf of the Symposium Team

\section{The 18th Bangkok International Symposium on HIV Medicine, Queen Sirikit National Convention Centre, Bangkok, Thailand, 13-15 January 2016}

Consistent with HIV-NAT's mission, quality training is provided to many professional healthcare workers in the region by taking the latest knowledge from research and presenting it locally at the Bangkok International Symposium of HIV Medicine. The symposium is offered every third week of January for 3 days. Some of the plenary session content is presented below.

First draft submitted: 29 January 2016; Accepted for publication: 2 February 2016;

Published online: 23 March 2016

\section{Special lecture}

- Second Professor Joep Lange \& Jacqueline van Tongeren Memorial Lecture: cost-effective strategies to end AIDS

Debbie Birx (Coordinator of the United States Government Activities to Combat HIV/AIDS and US Special Representative for Global Health Diplomacy, USA) thanked Thailand for being the forefront of HIV research and being willing to take risks. For example, Thailand undertook to work on the RV144 vaccine study even though Nobel Laureates stated that there were little data to proceed [1]. She commended Thai scientists and politicians for looking at its epidemic with a critical eye, and allowing data to drive its science and programmatic responses in the most optimal way (e.g., 100\% condom program, prevention of mother-to-child HIV transmission and HIV research collaboration models). Real-life experiences (data and models) from Thailand are now being used in Africa, with modifications to accommodate cultural and epidemic differences. However, she pointed out that there are some items that are hard to change in Africa, such as the frequency of

'HIV-NAT, Thai Red Cross - AIDS Research Centre, 104 Ratchadamri Rd, Pathumwan, Bangkok 10330, Thailand

2Division of Allergy \& Clinical Immunology, Department of Medicine, Faculty of Medicine, Chulalongkorn University, Rama IV Road, Pathumwan, Bangkok 10330, Thailand

${ }^{3}$ Division of Infectious Diseases, Department of Medicine, Faculty of Medicine, Chulalongkorn University, King Chulalongkorn Memorial Hospital, Rama IV Road, Pathumwan, Bangkok 10330, Thailand

${ }^{4}$ EATG in Europe (European AIDS Treatment group), Place Raymond Blyckaerts, 13, B-1050 Brussels, Belgium

${ }^{5}$ Thai Red Cross - AIDS Research Centre (TRCARC), 104 Ratchadamri Rd, Pathumwan, Bangkok 10330, Thailand

*Author for correspondence: Tel.: +66 2652 3040; Fax: +66 225 2579; juneohata4@gmail.com

\section{KEYWORDS}

- Asia $\bullet$ coinfections

- combination antiretroviral therapy $\bullet$ comorbidities

- cost-effectiveness

- elderly $\bullet$ HIV

- pre-exposure prophylaxis 
CD4 tests per year after the patient has started treatment, which is a significant financial barrier to scaling-up [2].

\section{Prevention utilizing current technology \\ - Innovative information technology approaches in HIV}

This nonmedical topic was addressed for the first time at the symposium because of its potential in public health programs in the country and region. Adam's Love is the leading internet and social media-based outreach organization in Thailand, specifically dedicated to the men who have sex with men (MSM) and the (transgender) TG communities. It provides online information and counseling on HIV, a clinic and research project in partnership with the TRCARC [3]. Cofounder Tarandeep Anand shared how surveys conducted by Adam's Love demonstrated that such services were reaching MSM with high levels of risk. For example, in one online survey, $46.25 \%$ did not use condom during last anal sex, $47.2 \%$ never tested for HIV and $40.1 \%$ have been exposed to HIV risk in the last month. Adam's Love is currently campaigning for the recruitment of participants in the PrEP-30 study. This implementation science study will compare three HIV outreach and care approaches - each targeting a different subset of the Adam's Love's website users. The aim is to find the best strategy to reach undiagnosed individuals in this key population: reaching out at community 'hotspots' in Thailand and referring to community-run clinics; testing and treatment provided entirely online; a hybrid of online outreach and offline clinic-based care. Adam's Love has expanded into Indonesia and Malaysia under the name of TemanTeman.org, and in Taiwan more recently, working with a hospital that provides HIV care, and the largest MSM sauna on the island. He concluded by emphasizing the 'need to be cared for' frequently expressed by young $\mathrm{MSM} / \mathrm{TG}$ in a qualitative survey on e-health interventions, and that Internet of Things was no longer a 'future trend,' because 'it has already happened.'

\section{Foreseeable HIV modalities}

- Future of HIV treatment \& care

David A Cooper (Kirby Institute, University of New South Wales, Australia) projected that we will continue to use non-nucleoside reversetranscriptase inhibitors sparing regimens for the next 5 years in developed countries. Tenofovir alafenamide will likely play a central role because it has fewer renal and bone side effects compared with tenofovir disoproxil fumarate (TDF) [4,5], and is to be given together with an integrase inhibitor as its anchor drug. It is highly likely that dolutegravir will replace efavirenz (EFV) [6]. It is also foreseeable that in the future, we will use longacting depot antiretroviral therapy (ART) [7,8]. However, until then, he emphasized the importance of using effective prevention strategies, such as $\operatorname{PrEP}[9,10]$ and male circumcision, with effective linkage to care utilizing civil societies to facilitate roll-out and reduce HIV incidence in order to achieve $90-90-90$ by 2020 .

\section{Morbidities}

- HIV \& aging: update \& practical approach

With the use of combination antiretroviral therapy, many HIV-infected people are living longer and experiencing comorbidities similar to nonHIV-infected populations. Treatment and care management among the elderly is difficult and unique. Victor Valcour (University of California, CA, USA) pointed out that in the treatment for HIV-infected elderly it is important to assess the 'root cause' of the problems. Management is complicated by multiple comorbidities and polypharmacy. He highly recommended treating patients by an intgerated multidisciplary team that could offer wholistic care to manage this rapidly expanding population.

\section{Roll-out strategies from a developing country's perspective \\ - Smart use of first-line in developing countries}

For this region, Kiat Ruxrungtham (Chulalongkorn University, Thailand) suggested optimizing doses as an approach to make best use of the current HIV modalities available. For example, lower doses of $400 \mathrm{mg}$ EFV in combination with tenofovir (TDF) and emtricitabine (FTC) in ART-naive adults with HIV-1 has been shown to be noninferior to the standard dose of $600 \mathrm{mg}$ at 48 and 96 weeks [11-13]. As a result of this, Thailand continues to prefer to use nonnucleoside reverse-transcriptase inhibitors, particularly EFV, as its first-line therapy because of its potency, efficacy, availability and cost [14]. The Thai Government Pharmaceutical Organization (GPO) is currently developing single tablet regimen of $400 \mathrm{mg}$ EFV with TDF/FTC, which can be conveniently administered once daily. If EFV cannot be tolerated, then it can be switched to rilpivirine [15,16]. 
Notable basic science lecture

- Immune activation \& inflammation in HIV \& clinical implications

Anthony Kelleher (Kirby Institute, University of New South Wales, Australia) elaborated on the immune response and immune activation. This extremely insightful plenary explained the benefits of starting ART early and the consequences for the immune system if treatment was deferred. When treatment is delayed, the thymus and lymph node tissues are damaged and have limited capacity to regenerate. This contributes to poorer clearance of various other infections such as $\mathrm{HCV}, \mathrm{HBV}$, CMV and HPV. However, even on ART, there is an increase in immune activation that results in morbidities, but earlier ART can decrease these events. Unfortunately, currently there are no clinically useful biomarkers and no drug interventions that have been shown to reduce immune activation. The most effective strategies for reducing complications arising from immune activation are lifestyle modifications (e.g., smoking cessation and exercise), treating clinically significant metabolic abnormalities, such as hypertension and diabetes, starting ART early, and treating coinfections (e.g., HBV, HCV).

\section{- The Great Debate: not offering PrEP to persons at high risk for HIV is unethical}

The debate was facilitated by Jintanat Ananworanich (US Military HIV Research Program). She clarified the definitions to be used in the debate: PrEP was any regimen, 'offer' was defined as to provide and recommend, high risk population meant adolescents and adults or any gender who engage in sexual and/or injecting behaviors that put them at an elevated risk for HIV infection, and 'unethical' was defined as not morally correct. The 'Pro' team, comprised Donn Colby (SEARCH, TRCARC, Thailand) and Niwat Suwanpatthana (National Community Advisory Board Thailand), and provided a list of 'myths', which they correctly perceived the other team would use to argue why PrEP should not be offered to high-risk populations. These included cost, side effects, development of drug resistance, difficulties in scheduling PrEP before and after sex, and lack of capacity in the system to accommodate the service. They emphasized that PrEP works and provided abundant research evidence (i.e., iPrEX, PROUD and IPERGAY) to support their statement, even noting a manuscript that one of the opposing debaters had published, recommending the use of PrEP for the European guidelines [17]. Nevertheless, the 'Con' team, represented by Juergen Rockstroh (University of Bonn, Germany) and Narin Hiransuthikul (Chulalongkorn University, Thailand) won the debate based on the technicality that data from clinical trials do not represent real-life situations and not all high-risk populations are ready to adhere to PrEP, so this is not the right time yet to implement it. The 19th Bangkok International Symposium on HIV Medicine will be held on 18-20 January 2017.

\section{Acknowledgements}

The authors would like to thank all of the chairpersons, speakers and moderators for their continued support in providing the most up-to-date information to the symposium. They are extremely grateful to all of their sponsors, collaborators and partners to make this event possible. The authors would also like to thank the participants who attended the event and provided feedback so they can work on improving the program. This symposium would not have been possible without the dedicated hard work and strong commitment from the Symposium Team for developing the scientific program to organizing, coordinating and running the show.

\section{Financial \& competing interests disclosure}

KRuxrungtham is the senior research scholar from Thailand Research Fund (TRF) and has received honoraria or consultation fees from Merck, Roche, Jensen-Cilag, Tibotec, Mylan and GPO (Governmental Pharmaceutical Organization, Thailand); and participated in a company-sponsored speaker's bureau from Abbott, Gilead, Bristol-Myers Squibb, Merck, Roche, Jensen-Cilag, GlaxoSmithKline and GPO. The authors have no other relevant affliations or financial involvement with any organization or entity with a financial interest in or financial conflict with the subject matter or materials discussed in the manuscript apart from those disclosed.

No writing assistance was utilized in the production of this manuscript.

\section{References}

1 Burton DR, Desrosiers RC, Doms RW et al. Public health. A sound rationale needed for Phase III HIV-1 vaccine trials. Science 303(5656), 316 (2004).
2 Manosuthi W, Ongwandee S, Bhakeecheep $\mathrm{S}$ et al. Guidelines for antiretroviral therapy in HIV-1 infected adults and adolescents 2014 Thailand. AIDS Res. Ther. 12, 12 (2015).
3 Anand T, Nitpolprasert C, Ananworanich J et al. How effective are innovative strategies that use communication technology in scaling up HIV testing and engaging MSM in HIV awareness? A case study from Thailand. Presented at: 8th IAS Conference on HIV 
Pathogenesis, Treatment and Prevention (IAS 2015). Vancouver, British Columbia, Canada, 19-22 July 2015.

4

Zolopa A, Ortiz R, Sax P et al. Comparative study of tenofovir alafenamide vs tenofovir disoproxil fumarate, each with elvitegravir, cobicistat, and emtricitabine, for HIV treatment. Presented at: 20th Conference on Retroviruses and Opportunistic Infections. Atlanta, GA, USA, 3-6 March 2013.

5 Pozniak A, Arribas J, Gupta SK et al. Safety of tenofovir alafenamide in renal impairment. Presented at: Conference on Retroviruses and Opportunistic Infections (CROI). Seattle, WA, USA, 23-26 February 2015.

6 Pappa K, Baumgarten A, Felizarta F et al. Dolutegravir (DTG) + abacavir/lamivudine once daily superior to tenofovir/emtricitabine/efavirenz in treatment-naive HIV subjects: 144-week results from SINGLE (ING114467). Presented at: 54th Interscience Conference on Antimicrobial Agents and Chemotherapy. Washington, D.C., USA, 5-9 September 2014.

7 Garcia-Lerma G. Monthly GSK744 long-lasting injections protect macaques against repeated vaginal SHIV exposures.
Presented at: 21st Conference on Retroviruses and Opportunistic Infections (CROI). Boston, MA, USA, 3-6 March 2014.

Margolis DA, Brinson CC, Smith GH et al. Cabotegravir and rilpivirine as 2-drug oral maintenance therapy: LATTE W96 results. Presented at: 22nd Conference on Retroviruses and Opportunistic Infections (CROI). Seattle, WA, USA, 23-26 February 2015.

9 McCormack S, D D. Pragmatic open-label randomised trial of preexposure prophylaxis: the PROUD study. Presented at: 22nd Conference on Retroviruses and Opportunistic Infections (CROI) Seattle, WA, USA, 23-26 February 2015.

10 Molina J, Capitant C, Spire B et al. On demand PrEP with oral TDF-FTC in MSM: results of the ANRS Ipergay Trial. Presented at: Conference on Retroviruses and Opportunistic Infections (CROI). Seattle, WA, USA, 23-26 February 2015.

11 Puls R, Amin J, Losso M et al. Efficacy of $400 \mathrm{mg}$ efavirenz versus standard $600 \mathrm{mg}$ dose in HIV-infected, antiretroviral-naive adults (ENCORE1): a randomised, double-blind, placebo-controlled, noninferiority trial. Lancet 383(9927), 1474-1482 (2014).
12 Carey D. Efavirenz $400 \mathrm{mg}$ daily remains non-inferior to $600 \mathrm{mg}$ : 96 week data from the double-blind, placebo-controlled ENCORE1 study. J. Int. AIDS Soc. 17(4 Suppl. 3), 19523 (2014).

13 Carey D, Puls R, Amin J et al. Efficacy and safety of efavirenz $400 \mathrm{mg}$ daily versus $600 \mathrm{mg}$ daily: 96-week data from the randomised, double-blind, placebo-controlled, non-inferiority ENCORE1 study. Lancet Infect. Dis. 15(7), 793-802 (2015).

14 Permpalung N, Putcharoen O, Avihingsanon A, Ruxrungtham K. Treatment of HIV infection with once-daily regimens. Expert Opin. Pharmacother. 13(16), 2301-2317 (2012).

15 European AIDS Clinical Society (EACS). Guidelines version 8.0, October 2015. www.eacsociety.org

16 British HIV Association (BHIVA). BHIVA Guidelines for the treatment of HIV-1-positive adults with antiretroviral therapy 2015. www.bhiva.org

17 Spinner CD, Boesecke C, Zink A et al. HIV pre-exposure prophylaxis $(\operatorname{PrEP})$ : a review of current knowledge of oral systemic HIV PrEP in humans. Infection doi:10.1007/s15010-0150850-2 (2015) (Epub ahead of print). 